permanence atrial fibrillation, the lower ratio of the hospitalisation for heart failures and improve the cardiac structure and function.

\section{GW23-e2529 THE FOLLOW-UP OBSERVATION FOR VVI AND DDD PACING IN SINUS BRADYCARDIA AFTER VALVE REPLACEMENT}

doi:10.1136/heartjnl-2012-302920a.103

Chen Min, Li Li, Xu Zhi yun, Chen Min. Department of Cardiothoracic Surgery,

Changhai Hospital, Second Military Medical University, Shanghai 200433, China

Objectives To compare the clinical effects of VVI and DDD pacing in sinus bradycardia in patients after valve replacement.

Methods All 29 patients with sinus bradycardia after valve replacement were divided into two groups by different pacing-mode. DDD group $(n=17)$, VVI group $(n=12)$. Mean follow-up was 28 months; Follow up the changes of left ventricular ejection fraction, fractional shortening and the size of atrial ventricular. The occurrence of permanence atrial fibrillation, the hospitalisation for heart failure, the stroke and the death.

Results All patients had no complications in Pacemaker implantation. There was none of stroke and death during the follow-up period. The incidence rate of permanence AF in DDD group was significantly lower than its in DDD group; The hospitalisation rate for heart failure in DDD group was significantly lower than its in VVI group. The left atrial volume and diameter VVI group was significantly increased compared with baseline, which were no significantly increased in DDD group;. The ejection fraction in VVI group was lower than that in DDD group $(p<0.05)$.

Conclusions DDD pacing-maker in the patient, who with sinus bradycardia after valve replacement, get the lower ratio of 